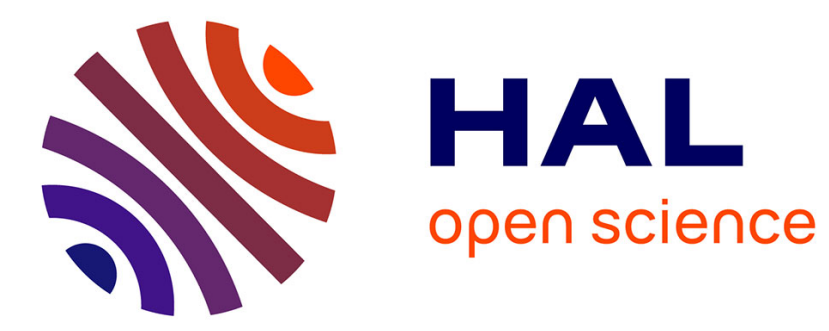

\title{
Solving constraints between lines in Euclidean geometry
}

Philippe Balbiani, Khalil Challita

\section{To cite this version:}

Philippe Balbiani, Khalil Challita. Solving constraints between lines in Euclidean geometry. 11th International Conference on Artificial Intelligence: Methodology, Systems, and Applications (AIMSA 2004), Sep 2004, Varna, Bulgaria. pp.147-157, 10.1007/978-3-540-30106-6_15 . hal-03252374

\section{HAL Id: hal-03252374 \\ https://hal.science/hal-03252374}

Submitted on 7 Jun 2021

HAL is a multi-disciplinary open access archive for the deposit and dissemination of scientific research documents, whether they are published or not. The documents may come from teaching and research institutions in France or abroad, or from public or private research centers.
L'archive ouverte pluridisciplinaire HAL, est destinée au dépôt et à la diffusion de documents scientifiques de niveau recherche, publiés ou non, émanant des établissements d'enseignement et de recherche français ou étrangers, des laboratoires publics ou privés. 


\title{
Solving constraints between lines in Euclidean geometry
}

\author{
Philippe Balbiani and Khalil Challita \\ Irit-CNRS, Université Paul Sabatier \\ 118 route de Narbonne, 31062 Toulouse Cedex 4, France \\ \{balbiani, challita\}eirit.fr
}

\begin{abstract}
We consider constraints satisfaction problems between lines in $\mathrm{Eu}-$ clidean geometry. Our language of constraints is based on the binary relation of parallelism. Our main results state that (1) solving constraints between lines in dimension 2 can be done in polynomial time whereas (2) solving constraints between lines in dimension 3 is NP-hard.
\end{abstract}

\section{Keywords}

Spatial reasoning - Constraint satisfaction problems - Euclidean geometry.

\section{Introduction}

The growing field of qualitative spatial reasoning finds its application in a variety of areas, such as Geographic Information Systems [9,10], Databases [18], Multimedia [23], Computer Vision and Artificial Intelligence. Most researchers have dealt with three main classes of spatial information. Topological relations describe how the interiors, the exteriors and the boundaries of two regions relate [3,7,9,20,21]. Directional relations describe the relative position of two regions to one another [11,14,24]. For instance, Ligozat [14] studies the algebra of cardinal relations between points in dimension 2. Distance relations describe the relative distance of two regions [10,27]. In artificial intelligence, variables usually denote points [5,6] or regions [20]. The latter alternative is more often preferred because a region takes into account the space that might be occupied by an object, and thus enables us to study a wider class of common problems. Affine geometry, one of the most prominent mathematical models of space, arises from the study of points, lines and planes by means of properties stated in terms of incidence. In coordinate geometry, lines are sets of points satisfying linear equations. Completely determined by two of their points, they can also be considered as abstract entities. They have certain mutual relations like, for instance, parallelism. Lines are to be found in many axiomatizations of affine geometry. However, we had great difficulty finding any examples of qualitative forms of spatial reasoning based solely on them. To confirm this claim, we have not been able to find any explicit reference to a qualitative spatial reasoning framework devoted to the study of lines in Euclidean geometry.

In this paper, we consider constraints satisfaction problems between lines in Euclidean geometry. Our language of constraints is based on the binary relation of parallelism. Our main results state that solving constraints between lines in dimension 2 can be 
done in polynomial time whereas solving constraints between lines in dimension 3 is NP-hard. The article is organized as follows. In section 2, we introduce relevant notions concerning constraint networks in general, and state some results about the consistency problem of constraints between lines in the Euclidean plane. Sections 3 and 4 generalize these results to a language enabling us to express ordering constraints or quantitative constraints between lines in dimension 2 . In section 5 , we establish that the complexity problem of networks of constraints between lines in dimension 3 is NP-hard, even for atomic ones.

\section{Lines in dimension 2.}

From now on, by $P O, E Q, D C$ we respectively mean: "having one point in common", "equal", "parallel and distinct". Two lines in the plane, denoted by $d$ and $d^{\prime}$, are exactly in one of the following relations: $d\{P O\} d^{\prime}, d\{E Q\} d^{\prime}, d\{D C\} d^{\prime}$. Let $E=$ $\{P O, E Q, D C\}$ be the set of the jointly exhaustive and pairwise disjoint relations that compare the position of any couple of lines in a plane.

The definitions needed for describing a constraint satisfaction problem (CSP), are defined by Montanari [17]. A network of linear constraints $\mathcal{R}$ is a couple $(I, C)$, where $I \subseteq I N$ is a finite set of variables, and $C$ is a mapping from $I^{2}$ to the set of the subsets of $E$ (i.e. $2^{E}$ ). The network $\mathcal{R}$ is atomic if for all $i, j \in I$, if $\operatorname{Card}(C(i, j))>1$ then $C(i, j)=E$. We say that $\mathcal{R}$ is path-consistent if for all $i, j, k \in I, C(i, j) \subseteq$ $C(i, k) \circ C(k, j)$. The composition table of the relations considered is given in figure 1 , where the symbols $<$ and $>$ account for the relation $\{D C\}$. A scenario (or an assignment) is a function $V$ that maps $I$ to a set of lines, in the Euclidean plane or in the Euclidean space. A scenario is consistent if for all $i, j \in I$, the relation that holds between the lines $d_{i}=V(i)$ and $d_{j}=V(j)$ is in $C(i, j)$. A relation $r \in C(i, j)$ is feasible if there exists a consistent scenario such that the lines $d_{i}$ and $d_{j}$ are in the relation $r$. A network of constraints is minimal if every relation in it is feasible. The notion of $k$ - consistency, adapted from Freuder [12], leads naturally to strong kconsistency, as defined by Vilain and Kautz [26]. We say that $\mathcal{R}$ is k-consistent if, given any consistent assignment of $k-1$ of its variables to lines in a Euclidean plane or Euclidean space, there exists a consistent assignment of any $k^{\text {th }}$ variable of the network. It is strongly $\mathrm{k}$-consistent if it is $\mathrm{j}$-consistent for all $j \leq k$. A network is said to be globally consistent if it is strongly n-consistent, for $n=\operatorname{Card}(I)$. Given a network of constraints, the question that arises is whether or not there is a consistent scenario for it. The algorithm of path consistency is explored and analyzed in $[15,16]$. The constraints propagation algorithm due to Allen [1], that replaces each constraint $C(i, j)$ by $C(i, j) \cap(C(i, k) \circ C(k, j))$, transforms in polynomial time each network $\mathcal{R}$ into a path-consistent one, whose set of consistent scenarios is the same as for $\mathcal{R}$.

Example 1. Path-consistent but not globally consistent network of linear constraints: $I=\{1,2,3,4,5\}$ and $C(1,2)=C(1,5)=C(3,4)=C(3,5)=\{P O, E Q\}$, $C(1,3)=C(1,4)=C(2,3)=C(2,4)=\{P O, D C\}, C(2,5)=\{P O, D C, E Q\}$, $C(4,5)=\{D C, E Q\}$. Indeed, if we consider its subnetwork where $C(1,2)=C(3,4)=$ $\{E Q\}$ and $C(1,3)=C(1,4)=C(2,3)=C(2,4)=\{D C\}$, we find it impossible 
to consistently assign the fifth variable. We can easily check that the above network is minimal.

The following result is an immediate corollary of proposition 2 (see below).

Proposition 1. The consistency problem of networks of linear constraints is decidable in polynomial time, with mean of a deterministic algorithm.

Consider a network $(I, C)$ of constraints between lines in the Euclidean plane. If this network is consistent then, obviously, we can find a consistent scenario $V$ such that for all $i$ in $I, V(i)$ is a nonvertical line in the Euclidean plane. $\left(a_{i}, b_{i}\right)$, where $y=a_{i} x+b_{i}$ is its algebraic equation. Indeed, as the constraint networks we consider are finite, we take care during the construction of a scenario $V$ not to assign to a variable $i$ a parallel line to the the $y$ axis, in order to avoid having equations of the form $x=c$, where $c \in I R$.

\section{Oriented parallelism}

From now on in this section, we only consider nonvertical lines in the Euclidean plane. It is a well-known fact that such lines are defined by a linear equation of the form $y=a x+b$ where $a$ and $b$ are real numbers. Given two parallel lines in the Euclidean plane, we give an orientation to their respective positions. Informally, for a line $d_{i}$, we wish to be able to distinguish the parallel lines that lie to its left from those that are to its right.

\begin{tabular}{|c|c|c|c|c|}
\hline$\circ$ & $\mathrm{EQ}$ & $<$ & $>$ & $\mathrm{PO}$ \\
\hline $\mathrm{EQ}$ & $\mathrm{EQ}$ & $<$ & $>$ & $\mathrm{PO}$ \\
\hline$<$ & $<$ & $<$ & $<,>, \mathrm{EQ}$ & $\mathrm{PO}$ \\
\hline$>$ & $>$ & $<,>, \mathrm{EQ}$ & $>$ & $\mathrm{PO}$ \\
\hline $\mathrm{PO}$ & $\mathrm{PO}$ & $\mathrm{PO}$ & $\mathrm{PO}$ & $<,>, \mathrm{EQ}, \mathrm{PO}$ \\
\hline
\end{tabular}

Fig. 1. Composition table of oriented 2D relations.

Definition 1. For any two lines $d_{i}$ and $d_{j}$ of the Euclidean plane with equations $y=$ $a_{i} x+b_{i}$ and $y=a_{j} x+b_{j}$ :

- $d_{i}\{<\} d_{j}$ iff $\left(a_{i}=a_{j}\right) \wedge\left(b_{i}<b_{j}\right)$.

- $d_{i}\{>\} d_{j}$ iff $\left(a_{i}=a_{j}\right) \wedge\left(b_{i}>b_{j}\right)$.

- An oriented $2 D$ network is a linear constraint network of lines in a Euclidean plane, where the relations are taken from the set $E=\{P O, E Q,<,>\}$. 
The binary relations $<$ and $>$ are the inverse of each other (i.e. $d_{i}\{<\} d_{j}$ iff $d_{j}\{>\} d_{i}$ ). We have the equivalence $d_{i}\{D C\} d_{j} \Leftrightarrow\left(\left(d_{i}<d_{j}\right) \vee\left(d_{i}>d_{j}\right)\right)$. The composition table of these relations is given in figure 1. We used the consistency-based composition of relations [4] to compute it: if $\alpha, \beta \in E$, then $\alpha \circ \beta$ contains all the relations $\gamma \in E$ such that there exists lines $d_{i}, d_{j}, d_{k}$ of the plane satisfying $d_{i}\{\alpha\} d_{j}, d_{j}\{\beta\} d_{k}$ and $d_{i}\{\gamma\} d_{k}$.

Example 2. Oriented 2D network which is not minimal: $I=\{1,2,3,4\}$ and $C(2,1)=$ $C(3,1)=C(4,1)=C(4,2)=C(4,3)=\{>, E Q\}, C(2,3)=\{<,>\}$. Indeed, the relation between the first and the fourth variables cannot be reduced to $\{E Q\}$.

The most important question about networks of constraints concerns the tractability of the consistency problem. The following proposition states that solving networks of constraints with oriented 2D relations can be done in deterministic polynomial time.

Proposition 2. The consistency problem of path-consistent oriented $2 D$ networks is in $P$.

Proof. In his work [14], and in order to compare the different positions of points $\left(a_{i}, b_{i}\right)$, and $\left(a_{j}, b_{j}\right)$ in the Euclidean plane, Ligozat defined the following nine relations: $(<,<),(<,=),(<,>),(=,<),(=,=),(=,>),(>,<),(>,=),(>,>)$. To each set $\Gamma$ of oriented 2D relations we can associate a set $\Gamma^{C}$ of cardinal relations such that an oriented 2D relation satisfied by the lines $d_{i}$ (with equation $y=a_{i} x+b_{i}$ ) and $d_{j}$ (with equation $y=a_{j} x+b_{j}$ ) is in $\Gamma$ iff the cardinal relation satisfied by the points $\left(a_{i}, b_{i}\right)$ and $\left(a_{j}, b_{j}\right)$ is in $\Gamma^{C}$ :

$\emptyset^{C}=\emptyset$.

$\{P O\}^{C}=\{(<,<),(<,=),(<,>),(>,<),(>,=),(>,>)\}$.

$\{E Q\}^{C}=\{(=,=)\}$.

$\{<\}^{C}=\{(=,<)\}$.

$\{>\}^{C}=\{(=,>)\}$.

$\{E Q,<\}^{C}=\{(=,=),(=,<)\}$.

$\{E Q,>\}^{C}=\{(=,=),(=,>)\}$.

$\{<,>\}^{C}=\{(=,<),(=,>)\}$.

$\{P O, E Q\}^{C}=\{(<,<),(<,=),(<,>),(>,<),(>,=),(>,>),(=,=)\}$.

$\{P O,<\}^{C}=\{(<,<),(<,=),(<,>),(>,<),(>,=),(>,>),(=,<)\}$.

$\{P O,>\}^{C}=\{(<,<),(<,=),(<,>),(>,<),(>,=),(>,>),(=,>)\}$.

$\{P O, E Q,<\}^{C}=\{(<,<),(<,=),(<,>),(>,<),(>,=),(>,>),(=,=),(=,<)\}$.

$\{P O, E Q,>\}^{C}=\{(<,<),(<,=),(<,>),(>,<),(>,=),(>,>),(=,=),(=,>)\}$.

$\{P O,<,>\}^{C}=\{(<,<),(<,=),(<,>),(>,<),(>,=),(>,>),(=,<),(=,>)\}$.

$\{E Q,<,>\}^{C}=\{(=,=),(=,<),(=,>)\}$.

$\{P O, E Q,<,>\}^{C}=\{(<,<),(<,=),(<,>),(>,<),(>,=),(>,>),(=,=),(=,<)$, $(=,>)\}$.

By construction, the consistency problem of an oriented 2D network is polynomially reducible to the consistency problem of a network of cardinal constraints. Moreover, we easily check that the sets of cardinal relations defined above are preconvex. We conclude our proof by recalling that the consistency problem of networks of preconvex cardinal constraints is in $P$ [14]. 


\section{Metric constraints in dimension 2}

Our aim in this section is to define quantitative relations that allow us to compare the distance that separates two parallel lines of the Euclidean plane. We will first consider relations that enable us to tell if, for two parallel lines $d_{i}$ and $d_{j}$, the distance between them (denoted later on by $d\left(d_{i}, d_{j}\right)$ ), is less or equal to a certain value. The latter relation will then be augmented by another one, allowing us to tell whether or not the distance from $d_{j}$ to $d_{i}$ is superior to a real number.

Definition 2. Let $h \geq 0$. For any couple $\left(d_{i}, d_{j}\right)$ of lines in the Euclidean plane:

- $d_{i}\left\{P_{h}^{+}\right\} d_{j} \Leftrightarrow d_{i}$ is parallel to $d_{j}$ and $d\left(d_{i}, d_{j}\right) \leq h$.

- $d_{i}\left\{P_{h}^{-}\right\} d_{j} \Leftrightarrow d_{i}$ is parallel to $d_{j}$ and $d\left(d_{i}, d_{j}\right) \geq h$.

- A metric 2D network is a linear constraint network of lines in a Euclidean plane, where the relations are of the form $\{P O\},\left\{P_{h}^{+}\right\}$and $\left\{P_{h}^{-}\right\}$.

Remarks. For $h>0$, consider two parallel lines in the Euclidean plane $\left(d_{i}, d_{j}\right)$, distant of $h$, which equations are $y=a_{i} x+b_{i}$ and $y=a_{j} x+b_{j}$. These lines are identical iff $d_{i}\left\{P_{0}^{+}\right\} d_{j}$. Moreover, $d_{i}\left\{P_{h}^{+}\right\} d_{j} \Leftrightarrow\left(\left(a_{i}=a_{j}\right) \wedge\left|b_{i}-b_{j}\right| \leq D_{a}\right)$, where the real $D_{a}$ satisfies the equation $h=\frac{D_{a}}{\sqrt{1+a_{i}^{2}}}$. Symmetrically, $d_{i}\left\{P_{h}^{-}\right\} d_{j} \Leftrightarrow\left(\left(a_{i}=a_{j}\right) \wedge\right.$ $\left.\left|b_{i}-b_{j}\right| \geq D_{a}\right)$. Hence, the constraints $\left\{P_{h}^{+}\right\}$and $\left\{P_{h}^{-}\right\}$are strictly equivalent to the constraints $\left\{\left[-D_{a}, D_{a}\right]\right\}$ and $\left\{\left[-\infty,-D_{a}\right] \cup\left[D_{a}+\infty\right]\right\}$. Thus we can invariably switch between $d_{i}\left\{P_{h}^{+}\right\} d_{j}$ and $\left(b_{i}-b_{j}\right) \in\left[-D_{a}, D_{a}\right]$ (the same reasoning applies to $d_{i}\left\{P_{h}^{-}\right\} d_{j}$ ). For the composition of relations of the form $\left\{P_{h}^{+}\right\}$and $\left\{P_{h}^{-}\right\}$(showed in figure 2), we use the definition of its counterpart, given in [8]: for any two intervals $T$ and $S$, the composition $T \circ S$ admits only values $r$ for which there exist $t \in T$ and $s \in S$, such that $r=t+s$.

\begin{tabular}{|c|c|c|l|}
\hline $\mathrm{O}$ & $\mathrm{P}_{\mathrm{h}}^{+}$ & $\mathrm{P}_{\mathrm{h}}^{-}$ & $\mathrm{PO}$ \\
\hline $\mathrm{P}_{\mathrm{r}}^{+}$ & $\mathrm{P}_{\mathrm{r}+\mathrm{h}}^{+}$ & $\mathrm{P}_{\mathrm{h}-\mathrm{r}}^{-}$ & $\mathrm{PO}$ \\
\hline $\mathrm{P}_{\mathrm{r}}^{-}$ & $\mathrm{P}_{\mathrm{r}-\mathrm{h}}^{-}$ & $\mathrm{P}_{\mathrm{R}}$ & $\mathrm{PO}$ \\
\hline $\mathrm{PO}$ & $\mathrm{PO}$ & $\mathrm{PO}$ & $\mathrm{PO}, \mathrm{P}_{\mathrm{R}}$ \\
\hline
\end{tabular}

Fig. 2. Composition of metric 2D networks' relations.

Let $h \leq 0$. For any line of the Euclidean plane $d_{i}$, the set of lines $d$ such that $d\left\{P_{h}^{-}\right\} d_{i}$ represents by convention all the parallel lines that are equal or parallel to $d_{i}$. In that case, the relation $\left\{P_{h}^{-}\right\}$will be denoted by $\left\{P_{R}\right\}$. Let us consider the inner difficulty of proving the consistency of a given metric $2 \mathrm{D}$ network of constraints.

Proposition 3. The consistency problem of path-consistent metric $2 D$ networks is NPcomplete. 
Proof. Given a metric 2D network $\mathcal{R}=(I, C)$, for all $i, j \in I$, let $P^{-}=\left\{P_{t_{1}}^{-}, \ldots, P_{t_{p}}^{-}\right\}$ and $P^{+}=\left\{P_{l_{1}}^{+}, \ldots, P_{l_{q}}^{+}\right\}$be the sets of all corresponding parallel constraints appearing in $C(i, j)$. It's clear that the disjunction of constraints $P^{-}$(resp. $P^{+}$) can equivalently be reduced to $P_{h_{i j}^{-}}$(resp. $P_{h_{i j}^{+}}$), where $h_{i j}^{-}=\min \left\{t_{k}: 1 \leq k \leq p\right\}$ (resp. $\left.h_{i j}^{+}=\max \left\{l_{k}: 1 \leq k \leq q\right\}\right)$. Let $\mathcal{R}^{\prime}=\left(I, C^{\prime}\right)$ be the constraint network obtained in this way. By replacing every occurrence of parallel constraint in $\mathcal{R}^{\prime}=\left(I, C^{\prime}\right)$ with the relation $\{D C\}$, we can polynomially check if the resulting network of linear constraints is consistent or not (due to proposition 1). If it is consistent, let $\mathcal{R}^{\prime \prime}=\left(I, C^{\prime \prime}\right)$ be a solution, which is an atomic linear constraint network. We then switch back to a subnetwork $\mathcal{R}_{\alpha}^{\prime}=\left(I, C_{\alpha}\right)$ of $\mathcal{R}^{\prime}$, where every relation $\{D C\}=C^{\prime \prime}(i, j)$ is replaced by the original parallel one (i.e. $P_{h_{i j}^{+}}$or/and $P_{h_{i j}^{-}}$). Thus, for all $i, j \in I$, either $C_{\alpha}(i, j)=\{P O\}$ or $C_{\alpha}(i, j) \in\left\{P_{h_{i j}^{+}}, P_{h_{i j}^{-}}\right\}$. Checking the consistency of $\mathcal{R}_{\alpha}^{\prime}$ leads us to find a consistent scenario for the variables $i, j \in I$, where $C_{\alpha}(i, j) \in\left\{P_{h_{i j}^{+}}, P_{h_{i j}^{-}}\right\}$. Knowing that the consistency problem of a TCSP is NP-complete [8], our proposition is proven.

Corollary 1. The consistency problem of path-consistent metric $2 D$ networks, where the only allowed parallel constraints are of the form $\left\{P_{h}^{+}\right\}$, is in $P$.

This result stems directly from the fact that the consistency problem of an $S T P$ (which is by definition an atomic $T C S P$ ) is in $P$ [8].

\section{Lines in dimension 3}

To compare the different positions of lines in the space, we add a fourth relation representing the non-coplanarity $(N C)$ between two lines. The set $E=\{P O, E Q, D C, N C\}$ contains relations that are jointly exhaustive and pairwise disjoint, where each one is equal to its inverse. We already know from the decidability of elementary geometry that the consistency problem of networks of linear constraints in the Euclidean space is decidable. We next show that this problem is NP-hard, by polynomially reducing the problem "Not-all-equal SAT" to the problem of lines in the space. The decision problem "Not-all-equal SAT" comes from classical propositional logic. Given a conjunction of clauses containing exactly 3 literals, the issue at stake is to determine whether there exists a valuation satisfying it and such that in no clause are all 3 literals equal in truth value. See $[19,22]$ for details.

Definition 3. A spatial network is a linear constraint network of lines in a Euclidean space.

As in section 3, we use the consistency-based composition of relations to compute the table in figure 3: if $\alpha, \beta \in E$, then $\alpha \circ \beta$ contains all the relations $\gamma \in E$ such that there exist lines $d_{i}, d_{j}, d_{k}$ of the space satisfying $d_{i}\{\alpha\} d_{j}, d_{j}\{\beta\} d_{k}$ and $d_{i}\{\gamma\} d_{k}$.

Example 3. Here are some examples of spatial networks, containing four elements. 


\begin{tabular}{|c|c|c|c|c|}
\hline$\circ$ & EQ & DC & PO & NC \\
\hline EQ & EQ & DC & PO & NC \\
\hline DC & DC & DC, EQ & PO, NC & PO, NC \\
\hline PO & PO & PO, NC & PO, NC, DC,EQ & PO, NC, DC \\
\hline NC & NC & PO, NC & PO, NC, DC & PO, NC, DC,EQ \\
\hline
\end{tabular}

Fig. 3. Composition table of spatial relations.

1. Non-consistent atomic spatial network: $C(1,2)=\{D C\}, C(1,3)=C(1,4)=$ $C(2,3)=C(2,4)=\{P O\}, C(3,4)=\{N C\}$. Indeed, we cannot consistently assign the fourth variable. This shows that the polynomial method of path consistency (Allen's triangulation) doesn't provide a complete decision procedure for the consistency problem of spatial networks.

2. Non-globally consistent spatial network: $C(1,2)=C(1,3)=C(2,3)=\{D C\}$, $C(1,4)=C(2,4)=\{P O\}, C(3,4)=\{N C\}$. If we assign to the third variable a line $d_{3}$ included in the plane defined by the parallel lines $d_{1}$ and $d_{2}$, we find it impossible to consistently assign the fourth one.

Proposition 4. The consistency problem of path-consistent spatial networks is NPhard.

Proof. Let $\phi=\left(C_{1} \wedge \ldots \wedge C_{n}\right)$ be a conjunction of $n$ clauses having exactly 3 literals. We next associate to $\phi$ a spatial network $\mathcal{R}_{\phi}$. For our purpose, $V A R=\{p, q, r, \ldots\}$ will designate the set of atoms appearing in $\phi$, and $\left\{d_{p}, d_{q}, d_{r}, \ldots\right\}$ their associated lines in $\mathcal{R}_{\phi}$. In the network $\mathcal{R}_{\phi}$, the lines $\left\{d_{0}, d_{1}, d_{0}^{\prime}, d_{1}^{\prime}, d, d^{\prime}\right\}$ that constitute our basic structure must satisfy the conditions: $d_{0}\{P O\} d_{1}, d_{0}^{\prime}\{P O\} d_{1}^{\prime}, d_{0}\{D C\} d_{0}^{\prime}, d_{1}\{D C\} d_{1}^{\prime}$, $d_{0}\{N C\} d_{1}^{\prime}, d_{1}\{N C\} d_{0}^{\prime}, d\{P O\} d_{0}, d\{P O\} d_{1}, d\{P O\} d_{0}^{\prime}, d\{P O\} d_{1}^{\prime}, d^{\prime}\{P O\} d_{0}^{\prime}$, $d^{\prime}\{P O\} d_{1}^{\prime}, d^{\prime}\{N C\} d$. For all $1 \leq i \leq n$, and for all atoms $p$ appearing in the clause $C_{i}=l_{i_{1}} \vee l_{i_{2}} \vee l_{i_{3}}$, we associate the lines $d_{p}$ and $d_{\neg p}$. These lines must satisfy the conditions: $d_{p}\{P O\} d_{0}, d_{p}\{P O\} d_{1}, d_{p}\{D C, P O\} d^{\prime}, d_{\neg p}\{P O\} d_{0}, d_{\neg p}\{P O\} d_{1}$, $d_{\neg p}\{D C, P O\} d^{\prime}$, et $d_{p}\{N C\} d_{\neg p}$.

The intuitive interpretation of the literals in $\phi$ is: for each literal $l \in\{p, \neg p: p \in$ $V A R\}, l$ is true if the line $d_{l}$ is included in the plane $(P)$, defined by the lines $\left(d_{0}, d_{1}\right)$, otherwise $l$ is false.

Notice that all lines $d_{l}$ are either parallel to $d^{\prime}$ and are included in $(P)$, or they intersect $d^{\prime}, d_{0}$ and $d_{1}$. We easily check that for each atom appearing in a clause $C_{i}$, the construction of the lines $d_{p}$ and $d_{\neg p}$ is mutually exclusive, in the sense that one of them is included in $(P)$, whereas the other passes through it.

To successfully complete our reduction, for each clause $C_{i}=l_{i_{1}} \vee l_{i_{2}} \vee l_{i_{3}}$, at least one line $d_{l_{i j}}$ must be included in $(P)$, and another one $d_{l_{i k}}$ must pass through it, where $(j, k \in\{1,2,3\})$. To achieve our aim, for all $1 \leq j \leq 3$, the lines $d_{l_{i j}}$ and $d$ must satisfy the following condition: $d_{l_{i j}}\{P O, N C\} d$. For each clause $C_{i}$, we represent the lines $d_{l_{i j}}$ in such a way that for every distinct $j, k, t \in\{1,2,3\}$, there exists $d^{\prime \prime}$ satis- 
fying $d_{l_{i j}}\{P O\} d^{\prime \prime}, d_{l_{i k}}\{P O\} d^{\prime \prime}$ and $d_{l_{i t}}\{N C\} d^{\prime \prime}$. This condition ensures that at most two lines are either parallel to $d^{\prime}$ or intersect it.

This proves that a formula $\phi$ is satisfiable iff $\mathcal{R}_{\phi}$ is consistent. As the reduction made above is done in polynomial time in the length of the formula $\phi$, the proposition is established. Hence, determining consistency of networks of constraints between lines in the Euclidean space is untractable. The following proposition implies that it is still untractable if one considers networks of atomic constraints.

Proposition 5. Any spatial path-consistent network is polynomially reducible to an atomic one.

Proof. Let $(I, C)$ be a spatial network. In order to prove this result, we need to check that for each $i, j \in I$ such that $C(i, j)$ is a disjunction of constraints of the set $E=$ $\{P O, E Q, D C, N C\}$, the variables $i, j$ and the constraint $C(i, j)$ can be equivalently replaced by an atomic spatial network. For all the ten cases stated below, we replace $C(i, j)$ by $\{P O, E Q, D C, N C\}$ and then add some relevant variables.

1. $d_{i}\{E Q, D C\} d_{j}$ : we add the variables $d_{u}, d_{v}$ together with the following constraints $\left(\left(d_{u}\{P O\} d_{v}\right) \wedge\left(d_{i}\{P O\} d_{u}\right) \wedge\left(d_{i}\{D C\} d_{v}\right) \wedge\left(d_{j}\{P O\} d_{u}\right) \wedge\left(d_{j}\{D C\} d_{v}\right)\right)$.

2. $d_{i}\{E Q, P O\} d_{j}$ : we add the variables $d_{u}, d_{v}, d_{w}$ together with the following constraints $\left(\left(d_{u}\{P O\} d_{v}\right) \wedge\left(d_{u}\{D C\} d_{w}\right) \wedge\left(d_{v}\{N C\} d_{w}\right) \wedge\left(d_{i}\{P O\} d_{u}\right) \wedge\left(d_{i}\{P O\} d_{v}\right) \wedge\right.$ $\left.\left(d_{i}\{P O\} d_{w}\right) \wedge\left(d_{j}\{P O\} d_{u}\right) \wedge\left(d_{j}\{P O\} d_{v}\right) \wedge\left(d_{j}\{P O\} d_{w}\right)\right)$.

3. $d_{i}\{E Q, N C\} d_{j}$ : we add the variables $d_{u}, d_{v}, d_{w}$ together with the following constraints $\left(\left(d_{u}\{N C\} d_{v}\right) \wedge\left(d_{u}\{N C\} d_{w}\right) \wedge\left(d_{v}\{N C\} d_{w}\right) \wedge\left(d_{i}\{P O\} d_{u}\right) \wedge\left(d_{i}\{P O\} d_{v}\right) \wedge\right.$ $\left.\left(d_{i}\{P O\} d_{w}\right) \wedge\left(d_{j}\{P O\} d_{u}\right) \wedge\left(d_{j}\{P O\} d_{v}\right) \wedge\left(d_{j}\{P O\} d_{w}\right)\right)$.

4. $d_{i}\{P O, N C\} d_{j}$ : we add the variables $d_{u}, d_{v}, d_{w}$ together with the following constraints $\left(\left(d_{i}\{P O\} d_{w}\right) \wedge\left(d_{j}\{P O\} d_{w}\right) \wedge\left(d_{w}\{N C\} d_{u}\right) \wedge\left(d_{i}\{P O\} d_{u}\right) \wedge\left(d_{j}\{P O\} d_{u}\right) \wedge\right.$ $\left.\left(d_{i}\{D C\} d_{v}\right) \wedge\left(d_{j}\{P O\} d_{v}\right)\right)$.

5. $d_{i}\{D C, N C\} d_{j}$ : we add the variables $d_{t}, d_{u}, d_{v}, d_{w}, d_{z}$ that respectively satisfy the same constraints as those satisfied by $d_{0}, d_{1}, d_{0}^{\prime}, d_{1}^{\prime}, d$, defined during the proof of proposition 4. Moreover, we impose the following conditions $\left(\left(d_{i}\{P O\} d_{u}\right) \wedge\right.$ $\left.\left(d_{i}\{P O\} d_{v}\right) \wedge\left(d_{i}\{N C\} d_{z}\right) \wedge\left(d_{j}\{P O\} d_{t}\right) \wedge\left(d_{j}\{P O\} d_{w}\right) \wedge\left(d_{j}\{N C\} d_{z}\right)\right)$.

6. $d_{i}\{D C, P O\} d_{j}$ : we add the variables $d_{u}, d_{v}, d_{w}$ together with the following constraints $\left(\left(d_{u}\{D C\} d_{w}\right) \wedge\left(d_{i}\{P O\} d_{w}\right) \wedge\left(d_{i}\{P O\} d_{u}\right) \wedge\left(d_{j}\{P O\} d_{w}\right) \wedge\left(d_{j}\{P O\} d_{u}\right) \wedge\right.$ $\left.\left(d_{v}\{N C\} d_{i}\right) \wedge\left(d_{v}\{P O\} d_{j}\right)\right)$.

7. $d_{i}\{E Q, D C, P O\} d_{j}$ : we add the variables $d_{u}, d_{v}$ together with the following constraints $\left(\left(d_{i}\{P O\} d_{u}\right) \wedge\left(d_{i}\{P O\} d_{v}\right) \wedge\left(d_{j}\{P O\} d_{u}\right) \wedge\left(d_{j}\{P O\} d_{v}\right) \wedge\left(d_{u}\{D C\} d_{v}\right)\right)$.

8. $d_{i}\{E Q, N C, P O\} d_{j}$ : we add the variables $d_{u}, d_{v}$ together with the following constraints $\left(\left(d_{i}\{P O\} d_{u}\right) \wedge\left(d_{i}\{P O\} d_{v}\right) \wedge\left(d_{j}\{P O\} d_{u}\right) \wedge\left(d_{j}\{P O\} d_{v}\right) \wedge\left(d_{u}\{N C\} d_{v}\right)\right)$.

9. $d_{i}\{D C, P O, N C\} d_{j}$ : we add the variables $d_{t}, d_{u}, d_{v}, d_{w}$ together with the following constraints $\left(\left(d_{u}\{P O\} d_{v}\right) \wedge\left(d_{v}\{D C\} d_{w}\right) \wedge\left(d_{u}\{N C\} d_{w}\right) \wedge\left(d_{t}\{P O\} d_{u}\right) \wedge\right.$ $\left(d_{t}\{P O\} d_{v}\right) \wedge\left(d_{t}\{P O\} d_{w}\right) \wedge\left(d_{i}\{P O\} d_{u}\right) \wedge\left(d_{i}\{P O\} d_{v}\right) \wedge\left(d_{i}\{N C\} d_{t}\right) \wedge\left(d_{j}\{P O\} d_{t}\right) \wedge$ $\left.\left(d_{j}\{P O\} d_{w}\right)\right)$.

10. $d_{i}\{D C, E Q, N C\} d_{j}$ : we add the variables $d_{u}, d_{v}, d_{w}$ together with the following constraints $\left(\left(d_{v}\{D C\} d_{u}\right) \wedge\left(d_{u}\{P O\} d_{w}\right) \wedge\left(d_{w}\{P O\} d_{v}\right) \wedge\left(d_{i}\{D C\} d_{u}\right) \wedge\right.$ $\left.\left(d_{i}\{N C\} d_{w}\right) \wedge\left(d_{j}\{D C, P O\} d_{u}\right) \wedge\left(d_{j}\{D C, P O\} d_{v}\right)\right)$. 
The path consistency property enables us to check that the resulting network has the same set of consistent scenarios as $(I, C)$. Also note that for a spatial network of $n$ elements, there are at most $n^{2}$ non atomic constraints. We conclude that our reduction is polynomial in the size of the network $(I, C)$, and is done in time $O\left(n^{2}\right)$.

Corollary 2. The consistency problem of path-consistent atomic spatial networks is NP-hard.

In the proof of proposition 4 , it suffices to transform the relations $\{P O, N C\}$ and $\{P O, D C\}$ into atomic ones.

\section{Conclusion and perspectives.}

In this paper we studied several path-consistent constraint networks of lines in the Euclidean plane and space. Our main goal was to determine the complexity of the consistency problem of a network of linear lines. We have already shown that for a network of linear lines, this problem is in $P$. Considering some special relations between two parallel lines in a plane, we established that the complexity problem of an oriented 2D linear network (i.e. when the orientation of parallel lines is taken into account) is still in $P$, whereas the one concerning a metric 2D network (i.e. when the distance separating two parallel lines is considered) is NP-complete. We then showed that the consistency problem of atomic networks of lines in the Euclidean space is NP-hard, by polynomially reducing the problem "Not-all-equal SAT" to the problem of lines in the space.

Our next step is to determine the complexity of oriented and metric networks in the Euclidean space. But first, an important question that arises is whether the consistency problem of spatial networks is NP-complete. Given such a network $\mathcal{R}$, we need to exhibit a non deterministic program running in polynomial time in the length of the spatial network's variables, that computes a consistent scenario for $\mathcal{R}$.

\section{Acknowledgements}

Khalil Challita benefits from a grant, allowed by the Lebanese national council for scientific research.

\section{References}

1. Allen, J., Maintaining knowledge about temporal intervals, Communications of the Association for Computing Machinery, 26, pp. 832-843, 1983.

2. Balbiani, P., Raisonner à propos des droites et des cercles: réseaux de contraintes et systèmes déductifs, Reconnaissance des Formes et Intelligence Artificielle (RFIA), 2004.

3. Bennett, B. Logical representation for automated reasoning about spatial relations, Ph.D. Thesis, School of Computer Studies, University of Leeds, 1997.

4. Bennett, B., Isli, A., Cohn, A.G. When does a composition table provide a complete and tractable proof procedure for a relational constraint language?, in: Proceedings of International Joint Conference on Artificial Intelligence (IJCAI), 1997. 
5. Clarke, B. A calculus of individual based on "connection", Notre Dame Journal of Formal Logic, 22, pp. 204-218, 1981.

6. Clarke, B. Individuals and points, Notre Dame Journal of Formal Logic, 26, pp. 61-75, 1985.

7. Cui, Z., Cohn, A., Randell, D. Qualitative and topological relationships in spatial databases in: Proceedings of SSD-93, pp. 296-315, 1993.

8. Dechter, R., Meiri, I., Pearl, J. Temporal constraint networks, Artificial Intelligence, pp. 6195, 1991.

9. Egenhofer, M.J. Reasoning about binary topological relationships, in: proceedings of SSD91, pp. 143-160, 1991.

10. Frank, A.U. Qualitative spatial reasoning about distances and directions in geographic space, Languages and Computing, pp. 343-371, 1992.

11. Freska, C. Using orientation information for qualitative spatial reasoning, in: Proceedings of COSIT-92, Lecture Notes in Computer Science, Springer, pp. 162-178, 1992.

12. Freuder, E. A Sufficient condition for backtrack-free search, Journal of the ACM, pp. 24-32, 1982.

13. Garey, M., Johnson, D. Computers and intractability, a guide to the theory of NPcompleteness, W. H. Freeman and Company, New York, 1979.

14. Ligozat, G. Reasoning about cardinal directions, Journal of Visual Languages and Computing, 9, pp. 23-44, 1998.

15. Mackworth, A. Consistency in networks of relations, Artificial Intelligence, pp. 99-118, 1977.

16. Mackworth, A., Freuder, E. The complexity of some polynomial network consistency algorithms for constraint satisfaction problems, Artificial Intelligence, pp. 65-74, 1985.

17. Montanari, U. Networks of constraints: Fundamental properties and application to picture processing, Information Sciences, pp. 95-132, 1974.

18. Papadias, D., Theodoridis, T., Sellis, T., Egenhofer, M.J. Topological relations in the world of minimum bounding rectangles: a study with R-trees, in: Proceedings in ACM SIGMOD-95, pp. 92-103, 1995.

19. Papadimitriou, C. Computational complexity, Addison Wesley, USA, 1994.

20. Randell, D., Cui, Z., Cohn, A. A spatial logic based on regions and connection, in Nebel, B., Rich, C., Swartout, W. (editors): Proceedings of the Third International Conference on Principles of Knowledge Representation and Reasoning. Morgan Kaufman, pp. 165-176, 1992.

21. Renz, J., Nebel, B. On the complexity of qualitative spatial reasoning: a maximal tractable fragment of the region connection calculus, Proceedings IJCAI-97, Nagoya, Japan, pp. 522$527,1997$.

22. Schaefer, T. The Complexity of satisfiability problems, in Conference Records of the Tenth Annual ACM Symposium on Theory of Computing, pp. 216-226, 1978.

23. Sistla, A.P., Yu, C., Haddad, R. Reasoning about Spatial Relations in Picture Retrieval Systems, in: Proceedings in VLDB-94, pp. 570-581, 1994.

24. Skiadopoulos, S., Koubarakis, M. Composing cardinal directions relations, in: Proceedings of the Seventh International Symposium on Spatial and Temporal Databases (SSTD'01), Lecture Notes in Computer Science, Springer, pp. 299-317, 2001.

25. Skiadopoulos, S., Koubarakis, M. Qualitative spatial reasoning with cardinal directions, in: Proceedings of the Seventh International Conference on Principles and Practice of Constraint Programming (CP'02), Lecture Notes in Computer Science, Springer, pp. 341-355, 2002.

26. Vilain, M., Kautz, H. Constraint propagation algorithms for temporal reasoning, Proceedings of the Fifth National Conference on Artificial Intelligence. American Association for Artificial Intelligence, pp. 377-382, 1986.

27. Zimmermann, K. Enhancing qualitative spatial reasoning-Combining orientation and distance, in: Proceedings of COSIT-93, Lecture Notes in Computer Science, Springer, pp. 69-76, 1993. 